\title{
Tecnologías digitales, creative commons y nuevos modos de producción cultural en Chile
}

\section{Digital technologies, creative commons and new modes of cultural production in Chile}

\section{Tecnologias digitais, Creative Commons e novos modos de produção cultural no Chile}

Andrés Maximiliano Tello, Universidad de Playa Ancha, Valparaíso, Chile (andres.tello@upla.cl)

\section{Consuelo Dinamarca Noack, Universidad de Chile, Santiago, Chile} (consuelodinamarcanoack@gmail.com)

Sebastián Escobar Pulgar, Universidad de Chile, Santiago, Chile (seba.escobarqt@gmail.com)

RESUMEN | Se presentan los resultados de una investigación cualitativa sobre los usos de las licencias creative commons en el campo cultural chileno. Desde 2005 hasta la fecha, estas herramientas se han expandido rápidamente en las nuevas formas de producción, circulación y consumo de obras en el campo cultural nacional. Con el fin de rastrear las distintas prácticas sociales vinculadas con el uso de estas tecnologías, nos enfocamos particularmente en las experiencias de los y las productoras culturales de sellos discográficos digitales y editoriales independientes. El estudio se basa en la información de veinte entrevistas en profundidad con agentes culturales de estos subcampos de producción que han inscrito sus obras con diferentes tipos de licencias. El análisis permite ahondar en los diferentes usos asociados a estas tecnologías y a nuevas dinámicas del campo cultural, caracterizadas por la creciente digitalización de sus prácticas y por la emergencia de ensamblajes socio-materiales que alteran los modos de producción cultural tradicionales. Muestra, además, que las estrategias de estos agentes culturales tienden a la conformación de prácticas alternativas a la mercantilización de la cultura y, al mismo tiempo, al cuestionamiento delas lógicas de legitimación dominantes en el campo cultural. Finalmente, se destaca la potencia política de modos de asociación e invención colectiva basados en la colaboración y no en la competencia.

PALABRAS CLAVE: tecnologías digitales; creative commons; netlabels; editoriales independientes; cultura. 
ABSTRACT/This article shows the results of a qualitative research on the uses of creative commons licenses in the Chilean cultural field. From 2005 to date, these tools have rapidly expanded into new forms of production, circulation, and consumption of works in the national cultural field. To trace the different social practices linked to the use of these technologies, we focus particularly on the experiences of cultural producers of netlabels and independent publishers. The study is based on information from twenty in-depth interviews with cultural agents from these subfields of production, who have registered their works with different types of creative commons licenses. The analysis of the interviews allows us to delve into the different uses associated with these technologies and new dynamics of the cultural field, characterized by the increasing digitalization of their practices and by the emergence of socio-material assemblages that alter traditional modes of cultural production. The study also shows that cultural agents' strategies tend to shape alternative practices to the commodification of culture and, at the same time, to question the dominant legitimation logics in the cultural field. Finally, from the analysis of the productive organization forms of these users of creative commons licenses in Chile, the political power of modes of association and collective invention based on collaboration and not on competition stands out.

KEYWORDS: digital technologies; Creative Commons; netlabels; independent publishers; culture.

RESUMO | Este artigo apresenta os resultados de uma pesquisa qualitativa sobre os usos de um determinado tipo de tecnologias digitais no campo cultural chileno: as licenças Creative Commons. Desde o ano de 2005 até hoje, essas ferramentas se expandiram rapidamente para novas formas de produção, circulação e consumo de obras no campo cultural nacional. Com o objetivo de traçar as diferentes práticas sociais vinculadas ao uso dessas tecnologias, nos concentramos principalmente nas experiências de produtores culturais de selos discográficos digitais (netlabels) e editoras independentes. O estudo é baseado em informações de vinte entrevistas em profundidade com agentes culturais desses subcampos de produção que registraram suas obras com diferentes tipos de licenças Creative Commons. A análise das entrevistas permite aprofundar os diferentes usos associados a essas tecnologias e as novas dinâmicas do campo cultural, caracterizadas pela crescente digitalização das suas práticas e pelo surgimento de agenciamentos sóciomateriais que alteram os modos tradicionais de produção cultural. Além disso, o estudo mostra que as estratégias desses agentes culturais tendem a conformar práticas alternativas à mercantilização da cultura e, ao mesmo tempo, questionar as lógicas de legitimação dominantes no campo cultural. Finalmente, a partir da análise das formas de organização produtiva desses usuários de licenças Creative Commons no Chile, destaca-se o poder político dos modos de associação e invenção coletiva baseados na colaboração e não na competição.

PALAVRAS-CHAVE: tecnologias digitais; Creative Commons; netlabels; editores independentes; cultura. 


\section{INTRODUCCIÓN}

Durante las primeras décadas del siglo XXI, la acelerada expansión de las tecnologías digitales hacia diferentes dimensiones de la vida social ha implicado profundos cambios económicos, jurídicos y políticos, así como también una transformación radical de nuestros contextos socioculturales y sus modos tradicionales de producción y distribución de conocimientos e información (Jenkins, 2008; Castells, 2009; Rifkin, 2014; Benkler, 2015; Van Dijck, 2016; Delfanti \& Arvidsson, 2019). Por lo mismo, podría afirmarse que las transformaciones tecnológicas recientes trascienden el espacio digital y afectan directamente a las múltiples formas de interacción, socialización e invención social contemporáneas. En ese sentido, como bien lo plantea la mirada sociológica de Saskia Sassen (2012, 2017), la emergencia de la llamada cultura digital se caracteriza por generar nuevas y complejas ecologías socio-materiales, es decir, nuevos ensamblajes sociotécnicos que incluyen también variables no-digitales — tales como la formación de subjetividades o las condiciones de trabajo de sus propios usuarios. De ahí que sea necesario preguntarse por quiénes tienen acceso a los distintos tipos de tecnologías digitales, así como también "qué se necesita para obtener acceso, y qué diferentes usuarios podrían extraerse de los dominios digitales existentes" (Sassen 2017, p. 72). En esa línea, aquí presentamos los resultados de un estudio exploratorio que se ha enfocado en los distintos usos y usuarios de una tecnología digital muy específica, las licencias creative commons (de ahora en adelante LCC), pero cuya amplia repercusión en las formas de producción cultural contemporáneas resulta difícil de ignorar.

Según un reciente informe de la propia organización Creative Commons, este tipo de herramientas digitales ha experimentado un notable incremento alrededor del mundo: si el 2006 el número de producciones culturales (música, libros, videos, fotografías, etc.) adscritas a estas licencias llegaba a los cincuenta millones en Internet, en 2017 el conjunto de obras licenciadas con LCC bordeaba ya los mil millones y medio (Creative Commons, 2018). Las LCC son al mismo tiempo un instrumento jurídico público - que otorga a cualquier usuario la posibilidad de reproducir, compartir y distribuir todo tipo de obras bajo diferentes condiciones, menos restrictivas que los tradicionales derechos de autor-y una tecnología más compleja, ya que asignan "etiquetas" a cada obra que "son después enlazadas a versiones legibles por máquinas de estas mismas licencias, las cuales permiten a las computadoras identificar automáticamente contenidos que se pueden fácilmente compartir" (Lessig, 2005, p. 227). A continuación nos proponemos ahondar en las transformaciones y los nuevos modos de uso que la introducción de estas licencias y sus tecnologías digitales asociadas generan en el campo cultural (Bourdieu, 2013; Bourdieu \& Wacquant, 2014) de nuestro país, concentrándonos especialmente 
en el ámbito de la edición independiente y en los sellos discográficos digitales (también llamados netlabels ${ }^{1}$.

\section{SURGIMIENTO DE LAS LICENCIAS CREATIVE COMMONS}

Los antecedentes de las LCC se vinculan con la emergencia del movimiento copyleft en el campo de la informática, a comienzos de la década de los ochenta, que tenía como principal objetivo promover la producción de software libre (Broussard, 2007; Busaniche, 2010; Frantsvog, 2012; Owen, 2014; Fuster Morell, Subirats, Berlinguer, Martínez, \& Salcedo, 2015). Producir códigos de software abiertos o libres implica básicamente convertir el diseño de programas informáticos en un tipo de producción accesible, transparente y susceptible de modificación y, por lo tanto, permitir la realización de mejoras progresivas de softwares mediante un trabajo cooperativo y voluntario entre distintos programadores y usuarios. De esa manera, el movimiento copyleft surge como reacción a la privatización del código fuente de los sistemas operativos, que impulsa el millonario negocio de la industria del software hasta el día de hoy. El desarrollo de licencias copyleft -y particularmente un tipo inicial de ellas, la General Public License (GNU) - se mostraría pronto como una suerte de modelo alternativo a la tradicional política del copyright (Vaidhyanathan, 2017), cuya expansión amenaza con codificar el conjunto de la producción cultural en Internet (Lessig, 2005; Busaniche, 2010; Frantsvog, 2012; Olwan, 2013; Owen, 2014).

Junto con la influencia del movimiento contra-cultural a favor del software libre, otro hito importante para la creación de las LCC fue la promulgación en 1998 de la Copyright Term Extension Act (CTEA) en Estados Unidos, que extendería los plazos de los derechos de autor, es decir, de los derechos patrimoniales restrictivos sobre obras literarias, artísticas o científicas, que desde entonces impiden que estas pasen a dominio público durante 70 años tras la muerte de su autor $y$, en el caso de una autoría corporativa, 120 años después de su creación. Durante el mismo año, se implementa también la Digital Millennium Copyright Act, ley estadounidense que endurece las penas para las infracciones de copyright en Internet, marcando la pauta que seguirán luego otros países alrededor del mundo (Lessig, 2005). Respondiendo a ese contexto, en 2001 un grupo de expertos en ciber-derecho y propiedad intelectual fundan en Massachusetts la organización sin fines de

1. El términonetlabel se refiere a los sellos discográficos que distribuyen su música principalmente en formatos digitales a través de Internet, principalmente en formatos como MP3, OGG o WAV. Por lo general, estos sellos enfatizan la distribución en línea de su música, mediante descargas gratuitas y LCC. Desde la década del 2000, los netlabels se han vuelto muy populares en la red. 


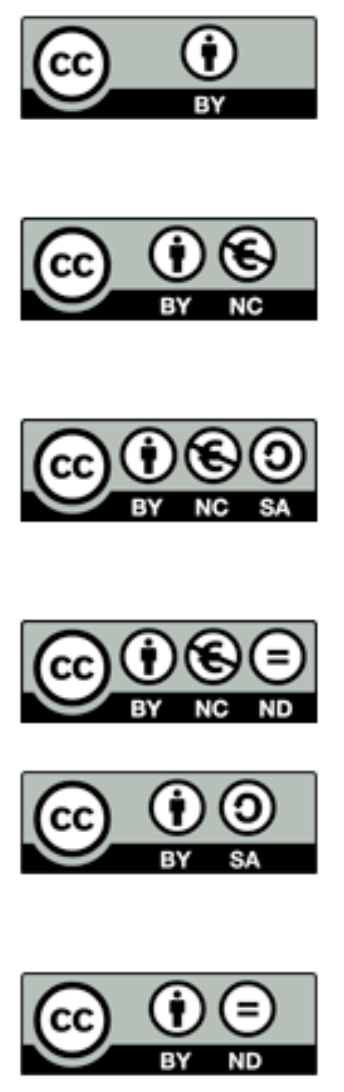

Reconocimiento (by): Se permite cualquier explotación de la obra, incluyendo una finalidad comercial, así como la creación de obras derivadas, la distribución de las cuales también está permitida sin ninguna restricción.

Reconocimiento - NoComercial (by-nc): Se permite la generación de obras derivadas siempre que no se haga un uso comercial. Tampoco se puede utilizar la obra original con finalidades comerciales.

Reconocimiento - NoComercial - Compartirlgual (by-nc-sa): No se permite un uso comercial de la obra original ni de las posibles obras derivadas, la distribución de las cuales se debe hacer con una licencia igual a la que regula la obra original.

Reconocimiento - NoComercial - SinObraDerivada (by-nc-nd): No se permite un uso comercial de la obra original ni la generación de obras derivadas.

Reconocimiento - Compartirlgual (by-sa): Se permite el uso comercial de la obra y de las posibles obras derivadas, la distribución de las cuales se debe hacer con una licencia igual a la que regula la obra original.

Reconocimiento - SinObraDerivada (by-nd): Se permite el uso comercial de la obra pero no la generación de obras derivadas.

Figura 1. Tipos de LCC

Fuente: Creative Commons.org

lucro Creative Commons, con el objetivo de desarrollar un conjunto de licencias alternativas que permitiese incrementar la creación de obras y su libre acceso en Internet, para "combatir la tendencia internacional hacia leyes de propiedad intelectual cada vez más restrictivas” (Olwan, 2013, p. 327).

Una de las figuras más destacadas tras la elaboración de las LCC, el abogado y académico estadounidense Lawrence Lessig, argumentará entonces que el avance del control sobre las obras que impulsan las leyes del copyright se convierte en una barrera para las dinámicas y el desarrollo de la propia cultura digital o, mejor dicho, en una amenaza para la "cultura libre" en Internet, aquella que deja "gran parte abierta a los demás para que se basen en ella”, posibilitando así la creación y la innovación sobre la base de las obras disponibles en nuestro acervo cultural (Lessig 2005, p. 38). A partir de ahí, la rápida expansión de las LCC en las producciones culturales difundidas en Internet se enmarca también en la gestación del llamado movimiento de la cultura libre (Lessig, 2005; Busaniche, 2010; Frantsvog, 2012; Fuster Morell et al., 2015). Actualmente, existen seis tipos básicos de LCC, cuya particular simbología funciona como etiqueta para su procesamiento digital y descripción jurídica específica, como muestra la figura 1. 
Estos seis tipos de licencias básicas son lanzadas por la organización Creative Commons el año 2002 en Estados Unidos (Lessig, 2005), y tres años más tarde fueron adoptadas en Chile. Desde entonces, un número ascendente de creadores nacionales (escritores/as, músicos/as, diseñadores/as, cineastas, etc.) optan por inscribir sus trabajos bajo esta forma alternativa de licenciamiento, lo que permite no solo una difusión mayor de sus obras en las plataformas digitales, sino que además otorga la posibilidad a productores/as y usuarios/as de compartirlas libremente (Cerda \& Ruiz, 2010; Gainza, 2018).

\section{CAMPO CULTURAL Y LICENCIAS CREATIVE COMMONS}

La masiva adopción de LCC en obras difundidas en Internet podría ser considerada como un importante rasgo de las prácticas de producción de bienes simbólicos en el campo cultural actual. De acuerdo con la teoría sociológica de Bourdieu (2013; Bourdieu \& Wacquant, 2014), las sociedades modernas se diferencian en campos sociales relativamente autónomos (campo político, económico, científico, religioso, jurídico, etc.), que desarrollan intereses, objetos y desafíos particulares. La estructura de cada campo social depende del estado de las relaciones de fuerza entre sus diversos agentes o instituciones, es decir, de la lucha por una posición hegemónica en el campo determinado y por la definición del capital simbólico específico para ese espacio. Cada campo impone así una visión y una división del mundo a aquellos que se involucran y aceptan tácitamente los presupuestos o reglas del juego de dicho espacio social (Albright \& Hartman, 2018). En el caso del campo cultural, las relaciones entre sus diferentes agentes o productores culturales dependen de la posición que ocupan "en la jerarquía que se establece bajo la relación de la legitimidad cultural en el interior del campo de las relaciones de producción y difusión de los bienes simbólicos" (Bourdieu, 2013, p. 135).

Ahora bien, la sociología de Bourdieu plantea que no todos los agentes pueden ocupar las mismas posiciones jerárquicas que estructuran el campo cultural, es decir, no todos los agentes alcanzan el mismo grado de consagración en el campo cultural (Bourdieu, 2013). La consagración opera principalmente en el "campo de producción restringida", que Bourdieu distingue del "sistema de gran producción simbólica", pues este último se ajusta principalmente a la demanda del mercado y, por lo tanto, tiene criterios comerciales, mientras que el primero debe entenderse como un "sistema que produce bienes simbólicos (e instrumentos de apropiación de estos bienes) objetivamente destinados (al menos a corto plazo) a un público de productores de bienes simbólicos que producen, también ellos, para productores de bienes simbólicos" (Bourdieu, 2013, p. 90). Así, el campo de producción restringida tiende a generar sus normas y criterios de evaluación de 
obras a partir del reconocimiento otorgado entre grupos de pares, es decir, entre otros agentes del campo cultural que resultan ser consumidores y competidores al mismo tiempo. Podríamos entonces preguntarnos si la masiva adopción de las LCC en el campo cultural contemporáneo altera de alguna manera este funcionamiento del campo de producción restringida al promover nuevos modos de producción y difusión de bienes simbólicos, permitiendo así que sus agentes desarrollen caminos alternativos a las tradicionales vías de legitimación cultural.

\section{METODOLOGÍA}

Para ahondar en las transformaciones de las dinámicas del campo cultural chileno vinculadas a la adopción de las LCC desde la década del 2000, nuestra investigación se focalizó en lo que podríamos denominar como una escena independiente dentro del campo de producción cultural, es decir, en aquellos productores y productoras culturales que inscriben (o han inscrito) sus obras bajo uno de los diferentes tipos de LCC. Además, nos concentramos particularmente en dos subcampos donde estas tecnologías digitales que permiten multiplicar los bienes comunes creativos se encuentran presentes en mayor medida: las editoriales independientes y los sellos discográficos digitales². Así, nuestro objetivo fue analizar, desde una perspectiva cualitativa, las prácticas, motivaciones y significaciones imbricadas en los distintos usos de las LCC por parte de los y las agentes culturales de editoriales independientes y netlabels nacionales. Para ello, se realizaron 20 entrevistas en profundidad, semiestructuradas, correspondientes a 10 agentes de editoriales independientes y 10 agentes de sellos digitales de la Región

2. Al mismo tiempo, cabe destacar que, de acuerdo con los datos del Observatorio de Políticas Culturales (OPC, 2016), el sector de la industria musical es uno de los que más ha crecido en los años recientes: entre 2005 y 2013 su crecimiento llegó a 536\%, sector donde además $82,5 \%$ corresponde a microempresas y $16,1 \%$ a pequeñas empresas. Según los datos del CNCA (2012-2014), 78\% de la producción musical se realiza en formato digital, y más de $60 \%$ de este corresponde a producciones culturales licenciadas con Creative Commons (Gainza, 2016, en OPC, 2016). Al igual que en el sector de la producción musical, durante la ultima década el sector editorial independiente se ha incrementado notoriamente, pues entre 2008 y 2014 se han fundado cerca de $83 \%$ de las editoriales de la escena independiente actual (Fuentes, Ferretti, Castro, \& Ortega, 2015). Por último, es importante resaltar que las editoriales independientes y los netlabels que constituyeron la muestra de este estudio producen obras principalmente en formato digital, aunque en algunos casos (principalmente en las editoriales) también publican obras en formato analógico o físico. Ahora bien, ya sea en el formato físico o digital, sus obras se licencian bajo creative commons. 
Metropolitana y la Región de Valparaíso, que son aquellas donde se concentra más del $80 \%$ de las y los productores culturales en Chile (Brodsky, Negrón, \& Pössel, 2014). El procedimiento utilizado para la selección de los y las informantes fue un muestreo bola de nieve, que logró reunir a veinte productores/as culturales que han registrado sus obras bajo cinco de los seis tipos de LCC: dos agentes culturales con LCC de Reconocimiento (BY); tres con LCC de Reconocimiento-No comercial (BY-NC); 11 con LCC de Reconocimiento-No comercial-Compartir igual (BY-NC-SA); tres con LCC de Reconocimiento-No comercial-Sin obra derivada (BY-NC-ND), y uno con LCC de Reconocimiento-Compartir Igual (BY-SA).

En ese sentido, la selección de los y las entrevistadas estuvo orientada por la necesidad de otorgar heterogeneidad a la muestra en cuanto a los tipos específicos de licenciamiento alternativo, pero también respecto de diferentes formas de producción cultural (en el caso de las editoriales independientes, se trata de publicaciones centradas en la narrativa, el pensamiento político, la poesía y la filosofía, mientras que en el caso de los netlabels se trata de sellos de música electrónica, experimental, rock, hip hop y folclore). Cada entrevista fue grabada, transcrita y codificada mediante el software AtlasTi 7.0, según temáticas claves asociadas a los objetivos de la investigación. Así, mediante el análisis de contenido cualitativo de la información recopilada, se ahondó en la particularidad de una escena del campo cultural nacional basada en la producción de bienes comunes creativos (Lessig, 2005), para trazar al mismo tiempo una radiografía descriptiva de los usos y tipos de usuarios de las LCC.

\section{RESULTADOS}

\section{Usos y usuarios de las LCC en la transformación digital del campo cultural}

La pregunta por los modos de uso de las tecnologías digitales, como en el caso de las LCC, debe dar cuenta de algo más que de la existencia de estas últimas y, por lo tanto, reconocer las "condiciones socio-materiales" de sus formas de acceso, que implican al mismo tiempo, la distinción de sus usuarios a partir de variables locales y contingentes (Sassen, 2017, pp. 72-73). En los casos que recogen las entrevistas de esta investigación, la mayoría de los modos uso de las LCC surgen de productores/ as culturales que difunden sus obras mediante sus propias editoriales y sellos digitales, cumpliendo distintas labores de forma simultánea (como la autoría de obras, su edición, gestión, difusión y distribución, entre otras). Esto quiere decir que los y las productoras culturales de netlabels y editoriales independientes se caracterizan por una marcada polifuncionalidad en sus labores. Al mismo tiempo, la mayor parte de las y los usuarios de las LCC asume su producción cultural como una forma de trabajo, es decir, esta actividad no se trata de un hobby o de 
un pasatiempo, sino de una labor donde se aspira a cierto profesionalismo: no se trata pues de "algo al vuelo, es algo que tiene un trabajo detrás... aunque sea gratis, también la idea es que no sea un chiste, que sea algo profesional" (Entrevista sello n 4). Ahora bien, los y las productoras culturales entrevistadas se reconocen en mayor medida como trabajadores independientes o por cuenta propia, lo que coincide con lo detectado en el catastro del trabajo cultural realizado por el Proyecto Trama, donde se establece que $56,6 \%$ de los productores culturales nacionales se encuentra en la misma condición (Brodsky et al., 2014)³. Además, la mayoría de los usuarios de LCC entrevistados posee estudios universitarios, otro trabajo remunerado que no se vincula con su labor cultural, y un rango etario predominante entre los veinte y los cuarenta años.

Dadas estas condiciones socio-materiales de producción, surge de inmediato la pregunta por la motivación tras la inscripción de sus obras bajo licencias que permiten una circulación abierta, lo que generalmente se traduce en una distribución gratuita de los productos de su trabajo cultural y, por lo tanto, sin una retribución monetaria a cambio. En este último punto habría que resaltar, en primera instancia, que una de las principales razones para el uso de las LCC esgrimida por las y los productores culturales entrevistados es de carácter fundamentalmente pragmático, es decir, su adopción de las LCC se deriva de la ejecución de su propia labor en la escena independiente. En otras palabras, el uso de las LCC les permite operar como productores/as culturales en distintos grados de independencia efectiva o de autogestión, respecto de las exigencias económicas, barreras geográficas u obstáculos institucionales relacionados con el registro de propiedad intelectual:
(...) el trámite básicamente tú lo haces desde tu casa, la inscripción, no tienes que ir a ningún lado, no tienes que gastar dinero en movilización, ni en pasajes, tú haces la inscripción desde tu casa y te da la opción que más te acomoda. Y tampoco hay que pagarle a las licencias Creative Commons para obtenerlas, tú las inscribes no más al momento, y eso es como bien automatizado. Cuando lo subes al sitio www.archive.org, donde nosotros tenemos alojada la música, ahí sale la opción; 'QQué tipo de licencias quiere?', y ahí uno se lo da cuando uno sube la música, viene como integrado, entonces se simplificó todo (Entrevista sello n³).

3. La relación entre nuevas formas de producción cultural y precarización del trabajo insinuada en este punto, y de la cual las entrevistas realizadas dan también cuenta, supone una reflexión mucho más profunda que no podemos desarrollar aquí, pero que esperamos desarrollar en una próxima publicación. 
Empecé a ocupar la licencia porque al vivir en Valparaíso me encontré con la problemática de que sacar la propiedad intelectual por Internet, tu hacías el depósito, la documentación que es muy sencilla, pero como sucede en todos los espacios que están centralizados, hay mucha burocracia, entonces era muy lenta la entrega del certificado con el número, y que no me permitía llevarlo a imprenta, ni terminar la tramitación del libro (Entrevista editorial nº 10).

El fácil acceso a las LCC, que permite eludir las barreras burocráticas e institucionales que las formas tradicionales de licenciamiento presentan, se muestra entonces como un elemento integrado a la propia labor de producción de esta escena independiente del campo cultural nacional. Sin embargo, la mayoría de los sellos digitales y las editoriales independientes entrevistadas coinciden en señalar que no solo las LCC son adoptadas por su accesibilidad, sino también porque permiten difundir de un modo más sencillo sus obras o producciones culturales a través de Internet, con un alcance nacional e internacional. Junto con esto, las entrevistas constatan que con "frecuencia es difícil para los creadores utilizar el trabajo de otro artista por las dificultades de encontrar al titular de los derechos y negociar una licencia”, en cambio, las LCC "facilitan que se compartan y distribuyan obras que de otro modo sería imposible", de modo que estas licencias "están revitalizando enormemente el flujo de información y creación" (Bollier, 2016b, p. 63). Por lo tanto, este vínculo entre las LCC y los entornos digitales resulta fundamental para analizar el desarrollo de la escena independiente que conforman los usuarios de las LCC dentro del campo cultural actual.

En ese sentido, las tecnologías digitales reconfiguran el campo cultural a comienzos del siglo XXI y se podría sostener que nos encontramos aquí con, al menos, dos transformaciones esenciales. En primer lugar, el amplio conjunto de "habilidades" y "competencias digitales" asociadas a los usos de las nuevas tecnologías pasa a convertirse hoy en una forma suplementaria de "capital simbólico", una suerte de "capital informacional" que permite a los agentes posicionarse de mejor manera dentro del campo cultural (Ragnedda, 2018). De hecho, es precisamente la incorporación y el manejo de las nuevas tecnologías digitales en su labor de producción independiente (uso de programas de edición, herramientas de diseño, creación y gestión de contenidos en páginas web, etc.) lo que permite a los netlabels y a las editoriales independientes constituirse como tales desde los recursos más mínimos (un computador y conexión a Internet), es decir, pese a no contar con la infraestructura y el equipamiento de las grandes industrias del rubro. Por ende, los y las productoras culturales de esta escena independiente recurren principalmente a la difusión en línea y a la autodistribución de sus obras, soslayando así los mecanismos de funcionamiento habituales de la gran industria editorial y discográfica, cuestión que coincide con lo señalado por otros estudios 
más generales sobre la configuración reciente de estas áreas del campo cultural nacional (Consejo Nacional de la Cultura y las Artes, 2014, pp. 174-197; Fuentes et al., 2015, p. 30; Observatorio de Políticas Culturales, 2016, p. 69).

Todos los productores/as culturales que conforman la muestra de este estudio cuentan con presencia digital, es decir, crean y gestionan contenidos en distintas plataformas digitales que funcionan con Creative Commons ${ }^{4}$, en sus propios sitios web o a través de sus distintas redes sociales. Esto nos lleva a un segundo cambio fundamental del campo cultural: el actual proceso de digitalización de las dinámicas de producción y difusión de bienes simbólicos posibilita la incorporación de nuevos agentes al campo de producción restringida (Bourdieu, 2013), quienes tienden a prescindir de los "intermediarios culturales tradicionales" (Pecourt \& Rius, 2018, p. 81). El uso de las LCC y otras tecnologías digitales permite entonces el surgimiento de una escena independiente que gira en torno a la cultura libre (Lessig, 2005), en donde las trayectorias de sus nuevos agentes no coinciden necesariamente con las formas de legitimación tradicionales (academias, conservatorios, crítica especializada, etc.), cuestión que genera ciertas tensiones o conflictos entre las y los productores consagrados y aquellos que son percibidos solo como aspirantes (Bourdieu, 2013). Como bien lo señala uno de los productores entrevistados, con la digitalización del campo cultural: "cualquiera puede ser músico... cualquiera en la casa con un computador puede ser director de un sello, hay gente a la que eso le parece súper bien y otra a la que le parece súper mal" (Entrevista sello n ${ }^{5}$ ). Desde luego, esta apertura del campo cultural propiciada por las tecnologías digitales no implica simplemente que todos los consumidores de literatura o de música se conviertan de inmediato en productores culturales, tal como lo sugieren visiones optimistas como la de la convergencia mediática (Jenkins, 2008); al contrario, la mayoría de los y las entrevistadas relata largos y arduos caminos recorridos para lograr cierto reconocimiento dentro de esa propia escena independiente.

Tal como lo demuestran las experiencias recogidas en esta investigación, si bien la inclusión de nuevos agentes en la escena independiente propiciada por la digitalización del campo cultural supone cierta democratización de este mismo, hay quienes sostienen que dicho proceso va de la mano con la erosión de la autonomía de las formas de producción cultural, que ceden su lugar frente a las estrategias

4. En el caso de los productores de obras musicales, algunas de las plataformas digitales que funcionan con LCC son Jamendo, Soundcloud, Audionity, FreeMusic Project, Vimeo y YouTube. Para el caso de las obras literarias, destacan particularmente las plataformas de fomento de la Cultura libre como Open Library, Online Computer Library Center, Internet Archive, entre muchas otras. 
de "autopromoción" y la "superficialidad" de contenidos que serían características de las plataformas digitales (Pecourt \& Rius, 2018, p. 82). Sin embargo, el aporte artístico y cultural de los sellos digitales y las editoriales independientes en el panorama artístico nacional difícilmente puede ser reducido.

Buena parte delosy las entrevistadas en este estudio ha logrado un reconocimiento de sus obras por parte de instituciones tradicionales del campo cultural (prensa especializada, revistas culturales, universidades, etc.), formando parte de una escena de usuarios de LCC que han contribuido a reactivar la producción cultural nacional a comienzos del siglo XXI (Observatorio de Políticas Culturales, 2016; Fuentes et al., 2015). No obstante, podría afirmarse que la escena independiente de la cultura libre configura también un nuevo campo de producción cultural digital, es decir, una escena cultural que produce bienes simbólicos destinados principalmente a un público en línea, que a su vez participa de la producción de obras. Este último se diferenciaría del "gran campo de producción simbólica" orientado exclusivamente a la conquista de un mercado (Bourdieu, 2013, p. 90). Se trata, en definitiva, de la reconfiguración de una escena alternativa dentro del campo de producción cultural digital, que se opone a la exclusiva valorización mercantil de las obras, funcionando al margen de la gran industria musical y del libro.

\section{El uso de las LCC como resistencia político-cultural}

Otro de los rasgos distintivos de este nuevo campo de producción cultural digital que conforman los usuarios de LCC en Chile es su recelo frente a las formas de licenciamiento tradicional de la propiedad intelectual que rigen el gran campo de producción simbólica, esto es, el de la producción cultural exclusivamente comercial. La mayoría de los y las entrevistadas rechaza registrar sus trabajos de esa manera, no porque estén en contra de las formas de reconocimiento o de atribución de una obra a un(a) autor(a), sino más bien porque, desde sus perspectivas, el copyright formaría parte de una gestión político-económica del trabajo artístico y, por extensión, del campo literario o musical, que afectaría de un modo negativo a las propias dinámicas de producción en medio del escenario digital, en tanto se convierte en un dispositivo de mercantilización de la cultura. A diferencia de esto último, los usuarios y usuarias de las LCC conciben sus propios modos de producción cultural como parte de una economía alternativa a la que atraviesa la lógica del mercado y la gran producción simbólica:

(...) escoger esa licencia era porque finalmente era eso; no tenía una atribución comercial, porque nosotros queríamos poner música para descarga gratuita, y también por el discurso que teníamos al menos en mis canciones o la gente que está como cercana, tenía sentido en el fondo hacerlo ellos mismos, entonces era no comercial... no podía ser de otra forma, y había cuestiones 
que se podían compartir, buenos hay documentales que hablan de eso. Pero, por ejemplo, si hay gente que quería hacer un remix o un matchup sobre eso, o construir a partir de eso samplear, esa opción también da la posibilidad, se supone que da esa posibilidad (Entrevista sello n 7).

La opción por un acceso abierto a las obras funciona entonces como un principio común de los modos de producción de los diferentes agentes de esta escena independiente del campo cultural digital. Los usuarios de las LCC aspiran a compartir sus obras con el mayor número de usuarios/as, abriendo en muchos casos la opción de modificar esos mismos trabajos o de convertirlos en insumos para la creación de nuevas obras. Al mismo tiempo, puede afirmarse que las LCC han promovido ciertos principios generales en estas nuevas formas de producción cultural, como el objetivo no-comercial de las obras, el rechazo a los derechos privativos del autor, y la liberación de los productos culturales. El acceso abierto se entiende aquí además como condición de posibilidad de la propia generación de cultura en el entorno digital; en ese sentido, el uso de metáforas como la del sampler o el remix podría describir no solo un tipo de trabajo artístico potenciado por las nuevas tecnologías, sino también el propio modo de funcionamiento que adquiere el trabajo creativo en nuestra época. Esto último es lo que remarca igualmente Lessig (2012) cuando sostiene que la expansión de internet hace florecer una "cultura remix", caracterizada por formas de trabajo creativo en las que la remezcla se produce en el seno de una nueva comunidad de remezcladores, cuyos miembros "en parte crean los unos para los otros, mostrándose mutuamente cómo saben crear" (Lessig, 2012, p. 111).

En ese sentido, la emergencia y proliferación de las LCC hace explícita la barrera que representan los derechos de autor para el trabajo creativo en Internet y en las plataformas digitales, cuestionando su viabilidad en un contexto de producción cultural profundamente transformado por las nuevas tecnologías de la información y de las comunicaciones (Lessig, 2012; Rifkin, 2014; Benkler, 2015). Las nuevas formas de trabajo creativo no se ajustan a las regulaciones del copyright, centradas en la restricción del acceso y la privatización de la cultura. Ahora bien, tal como lo plantea Siva Vaidhyanathan (2017), se trata aquí de un desajuste con toda una política del copyright, es decir, con un sistema complejo de interacciones entre diversas instituciones y agentes: organizaciones internacionales (partiendo por la Organización Mundial de la Propiedad Intelectual), gubernamentales (como el Instituto Nacional de Propiedad Intelectual o el Departamento de Derechos Intelectuales), legislaturas estatales, entidades de gestión de derechos de autor nacionales (como la Sociedad Chilena del Derecho de autor, SCD), "contratos del sector privado y los hábitos de escritores, artistas y músicos [que] influencian la manera en que opera el sistema del copyright" (Vaidhyanathan, 2017, p. XXXIII). 
Todas estas instituciones y agentes hegemónicos del campo cultural parecen entrar en conflicto con la nueva escena independiente vinculada a las LCC:

Las políticas de propiedad intelectual se rigen por estándares internacionales en Chile, ¿no? (...). Pero pienso que todo eso oculta la verdad sobre lo que es la cultura humana y de dónde emana y cómo se va creando, (...) eso es lo que nos importa tratar de recordar, cómo se ha formado la cultura humana y cómo se va creando, y pienso que estas políticas invisibilizan eso... tratan a la creación de la cultura de la misma manera en que tratan a cualquier otro recurso (Entrevista editorial no 4 ).

Con la idea de que el proceso de creación e innovación cultural se basa en una relación independiente del intercambio mercantil y ajena a una racionalidad economicista de los derechos de autor, se traza un imaginario del campo cultural digital como espacio fundamentalmente constituido por la compartición y el acceso abierto a las obras culturales. De ese modo, los agentes de la escena independiente parecen, en primera instancia, tener en común cierta mirada crítica sobre la producción de obras y la economía de la cultura en los entornos digitales. Como bien señala uno de los entrevistados al respecto: "la licencia, sin pedirte nada a cambio, además te permite a ti, al usarla, formar parte de una comunidad internacional de creadores, de productores culturales que comparten cierta visión, es una posición ideológica sobre la producción de elementos culturales" (Entrevista editorial n² 2 ). Sin embargo, dicha postura ideológica sobre los modos de producción cultural no es en ningún caso homogénea, porque si bien la mayoría de las visiones políticas de los y las entrevistadas podrían ubicarse dentro de lo que tradicionalmente se considera como una tendencia de izquierda, dichas posiciones oscilan dentro y fuera del amplio espectro de esta última.

Así, en las entrevistas encontramos a productores(as) culturales que se definen abiertamente como partidarios de una determinada ideología política, que atravesaría además el contenido de sus obras, pero también encontramos a quienes se desligan de cualquier posición política, aunque resaltan su postura crítica frente a la mercantilización de la cultura. Esto refuerza lo que se ha señalado respecto al movimiento de la Cultura Libre. En segundo lugar, pese a que en principio se podría suponer que la elección de cada una de las diferentes licencias respondería también a posiciones políticas diferenciadas, las posturas ideológicas de cada productor(a) cultural no se reflejan necesariamente en la elección de un tipo determinado de licencias; es decir, no hay una correspondencia entre las creencias y visiones del campo cultural de cada uno de los y las productoras y los distintos tipos de LCC que registran sus obras. De tal modo, por ejemplo, quienes optan por una licencia que exige compartir igual las obras derivadas —es decir, bajo la misma LCC-, no se 
diferencian sustancialmente en sus posturas de quienes registran sus trabajos con una LCC más restrictiva, como en el caso de aquella que no permite obras derivadas o impiden el uso comercial. Por lo tanto, el uso de un tipo específico de la gama de seis licencias por parte de las y los entrevistados no supone necesariamente posiciones políticas o planteamientos ideológicos determinados, más allá de un rechazo común a las políticas de privatización de la cultura o de expansión del copyright.

Ahora bien, pese a que la posición ideológica de la escena independiente no puede circunscribirse a una postura política exclusiva, la mayoría de los entrevistados coincide en sostener su rechazo a las políticas de derechos de autor, que verían asociadas al dominio de la gran industria cultural del libro y la música. Frente a la importancia comercial que las formas tradicionales de propiedad intelectual intentan instaurar en el escenario digital, las productoras/es culturales de nuestro estudio prefieren explorar más bien "la idea de las autorías más colectivas, o en las autorías que buscan ensamblarse o complementarse con otras" (Entrevista editorial no 8). Esto quiere decir que los usuarios de LCC rebaten la idea fetichizada de autoría, que ha convertido a la propiedad intelectual en "la mercancía ficticia por excelencia de nuestra época histórica” (Sádaba, 2008, p. 83). Los y las entrevistadas conciben al copyright como un marco jurídico en cuestionamiento y a las tecnologías ligadas a LCC como herramientas alternativas frente a la figura privativa que ejerce el registro de derechos de autor convencional. Esta oposición a los derechos privativos se conjuga con una concepción de la cultura como movimiento vivo, que fluctúa y se transforma en su propia circulación, y con una práctica organizativa que también entraña un modo de producción alternativo al de la cultura comercial. En ese sentido, la escena independiente implica nuevas formas de autogestión, cooperación y trabajo horizontal que constituyen simultáneamente formas de resistencia cultural, tal cual como las ha definido Stuart Hall (2017), pues el uso de las LCC está asociado aquí con diversas formaciones y fuerzas contrahegemónicas del establishment cultural y las políticas del copyright. La importancia de estas formas de resistencia cultural en el escenario digital no debe ser desconsiderada, pues como bien lo advierte Hall, ellas "crean la posibilidad de nuevas subjetividades", aunque lo cierto es que "no garantizan por sí mismas su contenido progresista o reaccionario. Para articularlas a posiciones políticas particulares aún hace falta apelar a prácticas sociales y políticas" (2017, p. 252).

\section{Creatividad común y tramas de organización sociotécnica}

El uso de las LCC ha permitido el desarrollo de nuevas modalidades de producción cultural marcadas por la colaboración y la asociatividad horizontal entre sus diversos agentes; asimismo, los diversos usos de las LCC contribuyen a reforzar la autogestión organizativa de los procesos de creación, promoción y difusión de obras en las editoriales independientes y los sellos digitales de nuestro país. En principio, los 
usuarios y usuarias de las tecnologías asociadas a las LCC parecen constituir lo que Jeremy Rifkin (2014) llama un "procomún social" que, a diferencia del mercado capitalista, "está motivado por el interés colaborativo y lo impulsa un deseo profundo de conectar y compartir con los demás. Si el primero fomenta el derecho de propiedad y la búsqueda de autonomía, el segundo promueve la innovación desinteresada, la transparencia y la creación de comunidad" (Rifkin 2014, p. 32). Los diversos usos de las LCC que caracterizan entonces a la escena independiente del campo cultural digital podrían insertarse dentro del amplio conjunto de prácticas sociales autogestionadas, cooperativas y descentralizadas que caracterizan también a las economías colaborativas de distintas comunidades de productores en el escenario digital, que manejan recursos colectivos y organizan alternativamente sus fuerzas creativas (Vercelli, 2010; Benkler, 2015; Bollier, 2016a). De acuerdo con las entrevistas realizadas, hay una clara coincidencia en los y las productoras de la escena independiente a la hora de señalar que las LCC "generaron una cooperatividad entre diferente gente" (Entrevista sello n 4 ), permitiendo de algún modo que "a los que nos interesa compartir, nos estemos encontrando" (Entrevista editorial no 4 ); es decir, las LCC funcionan como una tecnología aglutinadora de afinidades productivas entre distintos agentes del campo cultural digital. Esto es lo que refleja ejemplarmente uno de los entrevistados, desde la experiencia organizativa de un sello digital:

(...) generar una red de gente que trabajara bajo los mismo conceptos, es decir, si el sello distribuía música gratis, si el sello nunca pretendió tener auspiciadores o sponsor, o pedir que la gente donara o pagara por la música, también desde un principio dijimos: bueno, si este sello no es un negocio, la idea es que la relación entre los músicos tampoco sea un negocio. Y dijimos 'ya en este momento todos vamos a colaborar, todos vamos a poner su trabajo en forma gratuita con un objetivo común, que es levantar la plataforma y todo nos vamos a beneficiar unos con otros de lo que hacemos'. Por ejemplo, yo que era el diseñador hacia las carátulas para los discos, [otro amigo hacía] el mastering de los discos, tenía un amigo que era músico y profesor de inglés... hacia las traducciones de todos los comunicados, otro amigo que tenía parlantes ponía la amplificación para las fiestas, y así cada uno trabajaba por el sello en el sentido de que el objetivo era el mismo... y yo te diría que se creó esta red de amigos y la cosa se fue expandiendo (Entrevista sello n 5).

Los modos de producción de la escena independiente del campo cultural digital están atravesados por estas formas de cooperación y organización horizontal del trabajo. Sin embargo, habría que ser más cautos en este último punto, pues cierta perspectiva liberal (Benkler, 2015; Bollier, 2016a) asume que estos nuevos modos de producción cultural y sus formas de autoorganización funcionarían como complemento del mercado capitalista, al corregir de algún modo las falencias 
de sus mecanismos de competencia, fortaleciendo un sector productivo pero sin cuestionar su lógica de acumulación. Al contrario de dichas perspectivas, aquí se plantea que la dinámica de la escena independiente del campo cultural digital hace posible pensar "la cooperación social como el a priori de todos los procesos económicos, más que una forma particular entre otras o una reconciliación a posteriori de la vida económica y social" (Terranova, 2010, p. 19). En otras palabras, al demostrar que la cooperación es la base de cualquier proceso económico y no la iniciativa de actores individuales, este tipo de prácticas exhibe la potencia política de los procesos de invención colectiva y de generación de conocimiento común. Desde luego, esto implica rechazar el inmediatismo de las visiones más optimistas sobre el carácter político de las tecnologías digitales, que ven en Internet un espacio privilegiado para la democratización de la sociedad (Jenkins, 2008; Benlker, 2015), pero también distanciarse de las tesis más pesimistas sobre el movimiento de la Cultura Libre que sostienen que "Internet limita la cooperación y la crítica política, no las impulsa" (Rendueles, 2013, p. 56), generando una suerte de "individualismo tecnológico" (Rendueles \& Sádaba, 2009, p. 108).

A partir de los resultados de este estudio, es posible sostener que la radicalidad de las prácticas cooperativas de la escena independiente no puede apreciarse al considerar el uso aislado de las tecnologías digitales o de Internet, sino más bien atendiendo a la formación de sus complejos ensamblajes socio-materiales (Sassen, 2017). En dichos ensamblajes, las infraestructuras digitales forman parte de múltiples combinaciones con otras dimensiones analógicas y, por lo tanto, con otros tipos de objetos, técnicas, formas de interacción y organización social. En el caso de las editoriales independientes y los sellos digitales esto se hace evidente sobre todo en espacios como las ferias, las fiestas o las tocatas, donde las formas de interacción digital se articulan con el encuentro y la proxémica de los cuerpos, el protagonismo de los objetos analógicos (como libros y vinilos), las técnicas no digitales (como la empastación artesanal, el baile, etc.) y formas de cooperación emergentes o contingenciales, que no dejan de tramar vínculos societales más duraderos. De hecho, esta nueva escena independiente del campo cultural digital resulta impensable sin estos espacios de encuentro y sus diversas dimensiones sociotécnicas. Como bien lo plantea una de las entrevistadas:

Siento caleta $^{5}$ de alegría de que haya espacios donde se puedan compartir las cosas, y que haya gente que este en la misma de uno al final, porque ese es el espacio donde hacemos comunidad. Sipo ${ }^{6}$, las ferias emergentes, el espacio de la gente que no cree en el sistema establecido hace comunidad, y la gente

5. Expresión chilena que quiere decir mucho, bastante. Coloquial. (N.d.C.).

6. Versión coloquial de sí. (N.d.C). 
que tampoco se identifica quizás en las tendencias políticas más progresistas que son los que van a estos grandes escenarios culturales, o a estos grandes eventos culturales. Son espacios más pequeños, más acotados (Entrevista editorial $\left.n^{\circ} 1\right)$.

Por lo tanto, el uso de las tecnologías digitales por parte de los y las productoras culturales de la escena independiente apunta no solo a la libre circulación de la información o del acceso en línea a las obras, sino también a la generación de espacios alternativos de encuentro físico, donde se dan instancias de comercialización o trueque de obras y formas de cooperación comunitaria. Para las editoriales independientes, las ferias son los momentos en los que la venta de libros resulta más auspiciosa, mientras que, en el caso de los netlabels, las tocatas o fiestas son las instancias donde pueden recaudar ganancias y difundir su música en vivo. Ambos tipos de actividades dependen igualmente del uso que los y las productoras culturales hacen de las plataformas digitales, para difundir eventos y convocatorias, es decir, el empleo de las redes y los flujos de información digital genera otros espacios de encuentro no-digitales, ensamblajes socio-materiales donde del trabajo colaborativo que se produce en línea se complementa con formas de cooperación y trabajo presencial.

En ese sentido, las LCC son una tecnología y “una disposición de lo existente que hace lo real de cierta manera, que ha dinamizado prácticas culturales de resistencia a partir de los modos de vida dentro de los cuales se desarrolló (y que contribuyó a desarrollar)" (Ortiz \& Winik, 2012, p. 202). Esto último nos llevaría entonces a coincidir con las perspectivas que vinculan el desarrollo de las LCC no solamente con las cuestiones en torno a la propiedad intelectual, sino que además con la emergencia de un conjunto de prácticas cotidianas de existencia comunitaria al margen de las prácticas derivadas de la institucionalización de la propiedad privada y sus regímenes de apropiación de bienes materiales e inmateriales, cuestionando varias premisas de la economía clásica y de los paradigmas políticos tradicionales.

\section{CONCLUSIÓN}

La digitalización del campo cultural a comienzos del siglo XXI transforma las principales dinámicas y modos de producción de sus diversos agentes. Esto resulta claro en el caso de las editoriales independientes y los netlabels en Chile, especialmente en el uso que hacen los y las productoras culturales de tecnologías como las LCC, asociadas a un conjunto de nuevas habilidades y competencias digitales. Estas herramientas han permitido que las editoriales independientes y los sellos digitales puedan funcionar de manera autónoma, generando métodos de autogestión y canales de difusión alternativos. En ese sentido, las LCC han 
sido fundamentales para el surgimiento de nuevos agentes culturales y para la configuración de una escena emergente que, parafraseando a Bourdieu (2013), podríamos afirmar que opera como un nuevo campo de producción restringida digital, al rechazar las formas de valorización del trabajo artístico que predominan en los circuitos de la cultura comercial y la industria del entretenimiento. Al mismo tiempo, esta escena independiente comparte en sus usos de las LCC una crítica a las formas de privatización que las políticas del copyright llevan a cabo en el campo cultural, apostando por formas alternativas de licenciamiento y usos compartidos de las obras. Así, los usuarios de las LCC encarnan en sus modos de producción formas de resistencia cultural a los modelos productivos que se imponen en el mundo de la cultura, y que suponen la extensión de una lógica mercantilizadora sobre las fuerzas creativas colectivas.

Finalmente, es necesario remarcar que, si bien tecnologías digitales como las LCC promueven formas de cooperación y descentralización productiva mediante el trabajo en red, la formación de comunidades de productores que estas tecnologías impulsan no puede reducirse solamente a una dimensión digital. Los modos de producción de estos nuevos agentes culturales suponen más bien complejos ensamblajes sociotécnicos (Sassen, 2012, 2017), donde las tecnologías digitales son un elemento entre muchos otros aspectos no digitales. Esto se aprecia claramente en el caso de las editoriales independientes y los netlabels, cuya activa generación de espacios de encuentro como ferias, festivales o conciertos, es parte fundamental de una escena independiente que ha surgido, no obstante, bajo el alero del campo de producción cultural digital del siglo XXI. La organización de dichas instancias resulta fundamental para calibrar el potencial político de estos modos de producción de bienes comunes creativos, pues estas últimas demuestran que es la cooperación la que actúa como el motor de sus fuerzas inventivas y creativas.

\section{REFERENCIAS}

Albright, J. \& Hartman, D. (2018). Introduction: On Doing Field Analysis. In J. Albright, D. Hartman, \& J. Widin (Eds.), Bourdieu's Field Theory and the Social Sciences (pp. 1-15). Singapore: Palgrave Macmillan.

Benkler, Y. (2015). La riqueza de las redes. Cómo la producción social transforma los mercados $y$ la libertad (The Wealth of Networks. How Social Productions Transforms Market and Freedom). Barcelona, Spain: Icaria.

Bollier, D. (2016a). Pensar desde los comunes. Una breve introducción (Think like a Commoner. A short introduction of the life of the commons). Madrid, Spain: Traficantes de sueños.

Bollier, D. (2016b). El ascenso del paradigma de los bienes comunes (The rise of the commons good paradigm). In C. Hess \&E. Ostrom (Eds.), Los bienes comunes del conocimiento (Understanding Knowlegde as a Commons. From theory to practice) (pp. 51-64). Madrid, Spain: Traficantes de sueños. 
Bourdieu, P. (2013). El sentido social del gusto. Elementos para una sociología de la cultura (The Social Sense of Taste. Elements for a Sociology of Culture). Buenos Aires, Argentina: Siglo XXI.

Bourdieu, P. \& Wacquant, L. (2014). Una invitación a la sociología reflexiva (An invitation to reflexive sociology). Buenos Aires, Argentina: Siglo XXI.

Brodsky, J., Negrón, B., \& Pössel, A. (2014). El escenario del trabajador cultural en Chile (The Cultural Worker Scene in Chile). Santiago, Chile: Proyecto Trama/OPC.

Broussard, S. (2007). The Copyleft Movement: Creative Commons Licensing. Communication Research Trends, 26(3), 3-17. Retrieved from http://cscc.scu.edu/trends/v26/v26_n3.pdf

Busaniche, B. (Ed.). (2010). Argentina copyleft: la crisis del modelo de derecho de autor y las prácticas para democratizar la cultura (Argentina Copyleft: The Crisis of Copyright and Practices to Democratize Culture). Villa Allende, Argentina: Fundación Vía Libre.

Castells, M. (2009). Comunicación y poder (Communication and Power). Madrid, Spain: Alianza.

Cerda, A. \& Ruiz, C. (2010). Internet, Copyright y Derecho. Opiniones contingentes (Internet, Copyright and Law. Outstanding opinions). Santiago, Chile: ONG Derechos Digitales.

Consejo Nacional de la Cultura y las Artes (CNCA). (2014). Mapa de las industrias creativas en Chile. Caracterización y dimensionamiento (Chilean Creative Industries' Map. Characterization and Measurement). Santiago de Chile: CNCA.

Creative Commons. (2018). State of the Commons. Retrieved from https://stateof.creativecommons.org/

Delfanti, A. \& Arvidsson, A. (2019). Introduction to digital media. Hoboken, NJ: Wiley.

Frantsvog, D. (2012). All rights reversed: A Study of Copyleft, Open-Source, and Open-Content Licensing. Contemporary Issues In Education Research, 5(1), 15-22. https://doi.org/10.19030/cier.v5i1.6782

Fuentes, L., Ferretti, P., Castro, F., \& Ortega, R. (2015). La edición independiente en Chile. Estudio e historia de la pequeña industria (2009-2014) (Independent publishing in Chile. Small production's research and history (2009-2014)). Santiago, Chile: Cooperativa de Editores de la Furia.

Fuster Morell, M., Subirats, J., Berlinguer, M., Martínez, R., \& Salcedo, J. (2015). Procomún digital y cultura libre ¿Hacia un cambio de época? (Digital Commons and Free Culture. Towards a Change of Era?). Barcelona, Spain: Icaria.

Gainza, C. (2018). Narrativas y poéticas digitales en América Latina. Producción literaria en el capitalismo informacional (Digital narratives and poetics on Latin America. Literacy production on informational capitalism). Santiago, Chile: Cuarto Propio.

Hall, S. (2017). Estudios culturales 1983: una historia teorética (Cultural Studies 1983. A Theoretical History). Buenos Aires, Argentina: Paidós.

Jenkins, H. (2008). Convergence culture. La cultura de la convergencia de los medios de comunicación (Convergence culture. Where old and new media collide). Barcelona, Spain: Paidós. 
Lessig, L. (2005). Cultura libre. Cómo los grandes medios usan la tecnología y las leyes para encerrar la cultura $y$ controlar la creatividad (Free Culture. How Big Media uses technology and the law lo lock down culture and control creativity). Santiago, Chile: LOM.

Lessig, L. (2012). Remix. Cultura de la remezcla $y$ derechos de autor en el entorno digital (Remix. Making Art Commerce Thrive in the Hybrid Economy). Barcelona, Spain: Icaria.

Observatorio Políticas Culturales (OPC). (2016). La industria musical independiente en Chile. Cifras $y$ datos para una caracterización (Indie music industry in Chile. Data framing for a characterization). Santiago, Chile: OPC/IMICHILE.

Olwan, R. (2013). Intellectual Property and Development. Theory and Practice. Heidelberg, Germany: Springer-Verlag.

Ortiz, N. \& Winik, M. (2012). Políticas de los afectos. La cultura crítica entre licencias, ferias y libros independientes (Politics of affections. The critical culture between licenses, fairs and independent books). In S. Lago Martínez (Comp.), Ciberespacio y resistencias. Exploración en la cultura digital (Ciberspace and Resistances. An Exploration on Digital Culture) (pp. 197-212). Buenos Aires, Argentina: Hekht libros.

Owen, L. (2014). Selling Rights. London, UK: Routledge.

Pecourt, J. \& Rius, J. (2018). La digitalización del campo cultural y los intermediarios culturales: una crítica social del utopismo digital (Digitalization of the Cultural Field and Cultural Intermediaries: A Social Critique of Digital Utopianism). REIS. Revista Española de Investigaciones Sociológicas, (162), 73-90. https://doi.org/10.5477/cis/reis.162.73

Ragnedda, M. (2018). Conceptualizing Digital Capital. Telematics and Informatics, 35(8), 2366-2375. https://doi.org/10.1016/j.tele.2018.10.006

Rendueles, C. (2013). Sociofobia. El cambio político en la era de la utopía digital (Socio-phobia: Political Change in the Digital Utopía). Madrid, Spain: Capitán Swing.

Rendueles, C. \& Sádaba, I. (2009). Ciberfetichismo y cooperación (Cyber-fetichism and cooperation). In I. Sádaba (Ed.), Dominio abierto. Conocimiento libre y cooperación (Open Domain. Free Knowledge and Cooperation) (pp. 103-127). Madrid, Spain: Círculo de Bellas Artes.

Rifkin, J. (2014). La sociedad de coste marginal cero. El internet de las cosas, el procomún colaborativo $y$ el eclipse del capitalismo (The zero marginal cost society. The internet of things, the collaborative commons and the eclipse of capitalism). Barcelona, Spain: Paidós.

Sádaba, I. (2008). Propiedad intelectual ¿Bienes públicos o mercancías privadas? (Intellectual Property. Public Goods or Private Commodity?). Madrid, Spain: Los libros de la Catarata.

Sassen, S. (2017). Digital Cultures of Use and their Infraestructures. In J. Wajcman\& N. Dodd (Eds.), The Sociology of Speed. Digital, Organizational, and Social Temporalities (pp. 72-85). Oxford, UK: Oxford UniversityPress.

Sassen, S. (2012). Interactions of the Technical and the Social: Digital formations of the powerful and the powerless. Information, Communication \& Society, 15(4), 455-478. https://doi.org/10.1080/1369118X.2012.667912 
Terranova, T. (2010). Una altra vida: cooperació social i vida anorgànica (Another Life: social cooperation and a-organic life). Digithum, (12), 15-21. Retrieved from https:// www.raco.cat/index.php/Digithum/article/view/227618

Vaidhyanathan, S. (2017). Copyrights y copywrongs. El ascenso de la propiedad intelectual y su amenaza a la creatividad (Copyrights and Copywrongs. The Rise of Intelectual Property and How It Threatens Creativity). Bogota, Colombia: Ediciones Uniandes.

Van Dijk, J. (2016). La cultura de la conectividad: una historia crítica de las redes sociales (The Culture of Connectivity. A critical story of Social Media). Buenos Aires, Argentina: Siglo XXI.

Vercelli, A. (2010). Reconsiderando las tecnologías sociales como bienes comunes.

(Reconsidering Social Technologies as Common Goods). Íconos, (37), 55-64. Retrieved from http://hdl.handle.net/10469/2067

\section{FINANCIAMIENTO}

Este artículo se enmarca en el desarrollo del proyecto FONDECYT Iniciación No 11201122 y en el trabajo del proyecto csoc 02-1718, financiado por la Dirección General de Investigación de la Universidad de Playa Ancha.

\section{SOBRE LOS AUTORES}

ANDRÉS MAXIMILIANO TELLO, sociólogo por la Universidad de Concepción, magíster en Estudios Latinoamericanos por la Universidad de Chile y doctor en Filosofía por la Universidad de Salamanca y la Universidad de Valladolid, España. Académico del Departamento de Sociología de la Universidad de Playa Ancha, Valparaíso. Investigador responsable del FONDECYT Iniciación Nº 11201122.

https://orcid.org/0000-0002-6518-4619

CONSUELO DINAMARCA NOACK, socióloga por la Universidad de Playa Ancha y magíster en Estudios de Género y Cultura por la Universidad de Chile. Activista feminista y disidente porteña. Profesional de investigación proyecto PIASOC 180025 Mujeres y matemáticas en Chile. Docente de Trabajo Social en la Universidad Bolivariana. Docente en el Diplomado Género e Intervención Social, impartido por OTEC PRASOC.

iD https://orcid.org/0000-0001-7206-7085

SEBASTIÁN ESCOBAR PULGAR, sociólogo por la Universidad de Playa Ancha y estudiante de magíster en Comunicación Social por la Universidad de Chile. Profesional para el programa Quiero mi barrio (MINVU) en la Municipalidad de Maipú, Santiago de Chile. Docente en la carrera de Fotografía Profesional del Instituto Profesional ARCOS, sede Viña del Mar.

iD https://orcid.org/0000-0001-5009-6440 